\title{
A typical teratoid/rhabdoid tumor with leptomeningeal spread causing hydrocephalus occlusus
}

Christian Asel $^{1 *}$ and Franz A Fellner ${ }^{1,2}$

${ }^{1}$ Central Radiology Institute, Kepler University Hospital, Medical Faculty of the Johannes Kepler University, Linz, Austria

${ }^{2}$ Medical Faculty of the Friedrich-Alexander-University of Erlangen-Nürnberg, Erlangen, Germany

\section{Case report}

We report on an 8-month-old girl who recently had recurrent vomiting and ptosis on the left side. There has also been a lack of weight gain in the past few months. After an abdominal cause could be excluded clinically and sonographically in the external hospital, further sonography of the cerebrum was carried out. A hydrocephalus internus of unclear origin was hereby found. The girl was immediately referred to our center, where a quick clarification by MR imaging of the cerebrum was performed. This examination revealed a tumor in the area of the fourth ventricle, already with meningeal spread, as the cause of the hydrocephalus occlusus and the cranial nerve symptoms. The histogenetic analysis finally resulted in the diagnosis of an atypical teratoid/rhabdoid tumor (AT/RT) (Figures 1-4). After neurosurgical assessment, prompt operative care and subsequent chemotherapy were carried out.

\section{Discussion}

Atypical teratoid/rhabdoid tumors (AT/RT) are rare with a total of $1-2 \%$ of the pediatric tumors of the CNS. They reach the highest prevalence of $10-20 \%$ at the age of $2-3$ years [1-3]. Clinically, this WHO grade IV tumor usually presents with vomiting, lethargy, headache, ataxia, seizures, macrocephaly or cranial nerve symptoms. The location is approximately half supra- or infratentorial. A partially observed intraventricular spread mainly affects the fourth ventricle. Leptomeningeal seeding is also described in $15-30 \%$ of cases [1]. They present themselves CT-morphologically as iso- to hyperdense solid

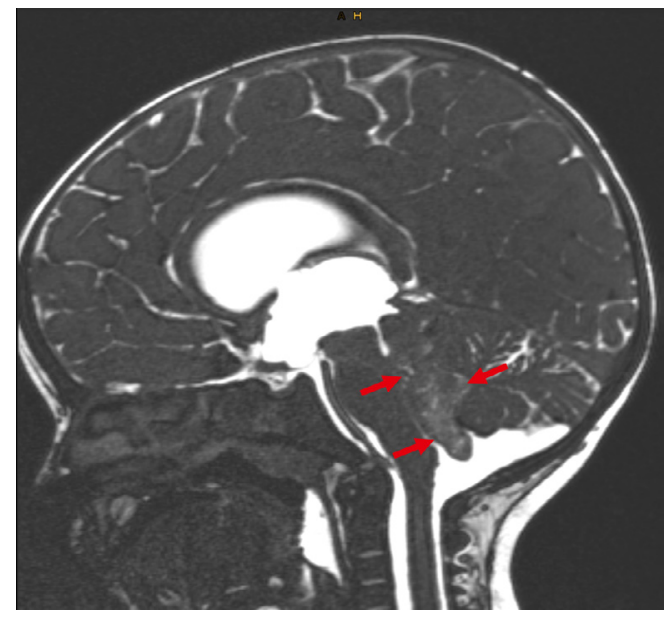

Figure 1. T2-weighted sagittal image. Tumor (AT/RT) in the IV ventricle with occlusion of the aqueduct (arrows) in an 8-month-old female patient

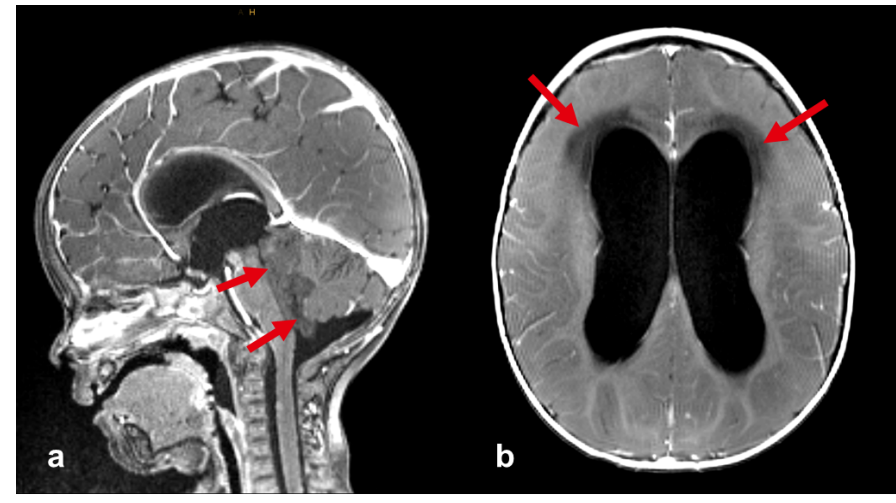

Figure 2. a) The T1-weighted 3D gradient-echo image after i.v. contrast administration shows the tumor (arrows) with occlusion of the 4 th ventricle and the resulting dilatation of ventricles 1-3. b) The T1-weighted contrast-enhanced spin-echo image reveals transpendymal edema (arrows) as a result of the occlusive hydrocephalus

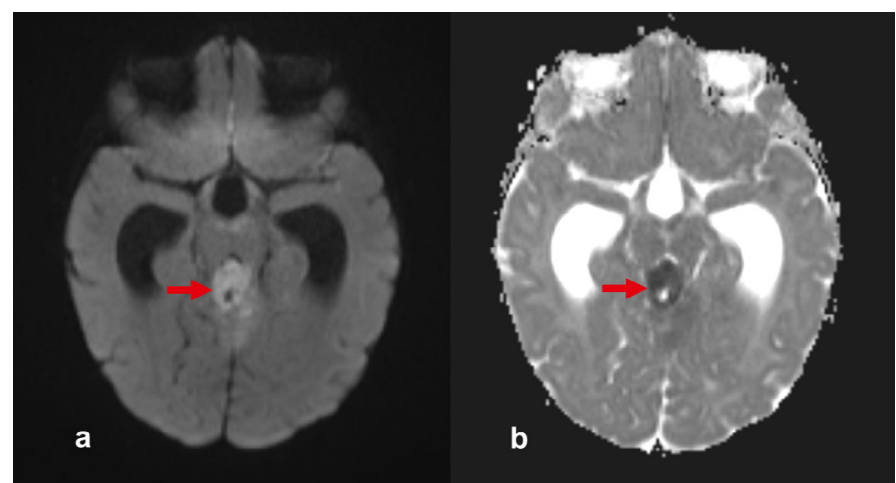

Figure 3. a) Diffusion-weighted image $(b=1000)$, b) ADC map. DWI/ADC. There is significant reduction of diffusion in this atypical teratoid/rhabdoid tumor

tumors, often with heterogeneous contrast enhancement, large tumor necrosis, bleeding and calcification. MR imaging reveals necrosis, multifocal cystic parts and bleeding. Accordingly, the tumor presents with different $\mathrm{T} 1$ and $\mathrm{T} 2$ signal behavior, depending on the amount of

*Correspondence to: Christian Asel, Central Radiology Institute, Kepler University Hospital, Medical Faculty of the Johannes Kepler University, Linz, Austria, E-mail: christian.asel@kepleruniklinikum.at

Key words: atypical teratoid/rhabdoid tumor (AT/RT), hydrocephalus, leptomeningeal spread, magnetic resonance $(M R)$ imaging

Received: April 03, 2020; Accepted: April 29, 2020; Published: May 04, 2020 


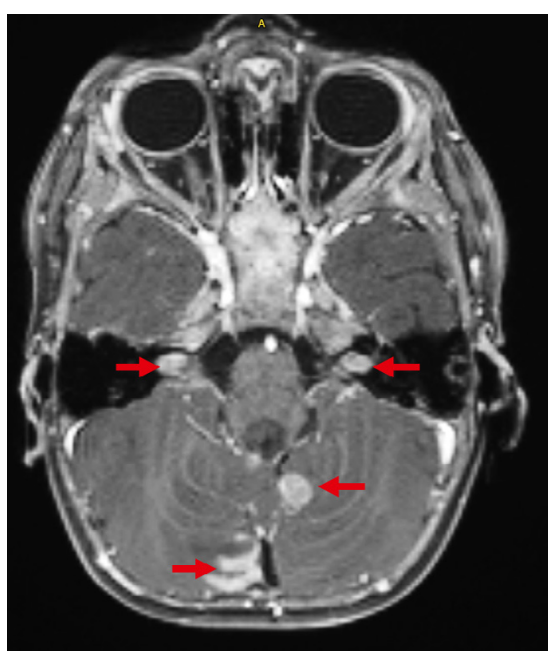

Figure 4. The T1-weighted spin-echo image after i.v. application of contrast agent demonstrates diffuse leptomeningeal tumorspreading (arrows)

hemorrhage in, contrast agent uptake or degree of calcification. Almost all AT / RT's have restricted diffusion [3]. In terms of differential diagnosis, this tumor entity is primarily opposed to medulloblastomas, ependymoblastomas, PNETs and plexus carcinomas. According to the WHO 2016 classification scheme, the definitive diagnosis requires proof of a special genetic deviation (loss of the INI1 tumor suppressor gene on chromosome 22 or BRG1 gene) [4]. There is no standardized therapy; radio chemotherapy usually follows the maximum possible tumor resection. The prognosis is extremely poor, and survival is reported to be $5-11$ months.

\section{Disclosure}

No conflicts of interest. No grants or financial support.

\section{References}

1. Nesvick CL, Nageswara Rao AA, Raghunathan A, Biegel JA, Daniels DJ (2019) Casebased review: atypical teratoid/rhabdoid tumor. Neurooncol Pract 6: 163-178.

2. PDQ Pediatric Treatment Editorial Board. Childhood Central Nervous System Atypical Teratoid/Rhabdoid Tumor Treatment (PDQ $\left.{ }^{\circledR}\right)$ : Patient Version. 2020 Mar 6. In: PDQ Cancer Information Summaries [Internet]. Bethesda (MD): National Cancer Institute (US); 2002-.2020

3. Katz JS, Peruzzi PP, Pierson CR, Finlay JL, Leonard JR (2017) Cerebellopontine angle tumors in young children, displaying cranial nerve deficits, and restricted diffusion on diffusion-weighted imaging: a new clinical triad for atypical teratoid/rhabdoid tumors. Childs Nerv Syst 33: 833-838.

4. Kohashi K, Oda Y (2017) Oncogenic roles of SMARCB1/INI1 and its deficient tumors. Cancer Sci 108: 547-552.

Copyright: (C2020 Asel C. This is an open-access article distributed under the terms of the Creative Commons Attribution License, which permits unrestricted use, distribution, and reproduction in any medium, provided the original author and source are credited. 